\title{
Modelagem do Processo de Fragmentação de Catalisadores Suportados Durante a Pré-polimerização de Olefinas
}

\author{
Douglas M. Merquior \\ Departamento de Engenharia Química, IME \\ Enrique L. Lima, José Carlos Pinto \\ Programa de Engenharia Química, COPPE, UFRJ
}

\begin{abstract}
Resumo: Uma metodologia é proposta para descrever a morfologia das partículas de polímero que são obtidas durante os momentos iniciais da polimerização de olefinas via catálise heterogênea. O método é baseado na análise matemática da capacidade da partícula em liberar a energia mecânica acumulada no seu interior devido à rápida produção de polímero. $\mathrm{O}$ balanço entre as quantidades de energia acumulada e liberada é calculado com o auxílio de um modelo dinâmico da reação de pré-polimerização. A combinação da metodologia proposta com o modelo dinâmico permitiu a análise dos mecanismos de fragmentação, indicando a morfologia da partícula de polímero produzida em função do tamanho da partícula e da temperatura do reator.
\end{abstract}

Palavras-chave: Fragmentação, catálise heterogênea, pré-polimerização, olefina.

\section{Modeling of Catalyst Fragmentation During Olefin Pre-polymerizations}

Abstract: A model-based methodology is proposed for describing the morphology of the polymer particles that are obtained during the very early stages of the olefin polymerization. The method is based on the analysis of the particle capacity to release the amount of energy that is accumulated in its interior during the polymerization, due to the fast polymer production. The balance between the accumulated and released amounts of energy is calculated with the help of a dynamic pre-polymerization reaction model. The combination of the fragmentation criteria and of the polymerization model allows the analysis of the prepolymerization step, indicating the morphology of the final polymer particles as a function of the catalyst particle diameter and reactor temperature.

Keywords: Fragmentation, heterogeneous catalysis, prepolymerization, olefin.

\section{Introdução}

$\mathrm{Na}$ indústria de polímeros, as poliolefinas ocupam posição de destaque pela quantidade de resina produzida, recursos movimentados ao redor do mundo e perspectivas promissoras de crescimento. Espera-se que as poliolefinas substituam diversos materiais plásticos atualmente em uso, cujos processos de manufatura ou reciclo sejam menos vantajosos ${ }^{[1]}$.

Para acompanhar este forte crescimento da de- manda por poliolefinas, diversos esforços têm sido feitos para melhorar a produtividade das plantas industriais, bem como aprimorar a qualidade das resinas olefínicas produzidas. Os catalisadores metalocênicos vêm atender estes dois aspectos, conjugando maior produtividade com a possibilidade de sintetizar polímeros com novas estruturas ${ }^{[1,2]}$. Entretanto, esta alta atividade contribui para a elevação do risco de instabilidade térmica, principalmente no início da reação. A fim de se aproveitar a maior produtividade

Autor para correspondência: José C. Pinto, Programa de Engenharia Química, COPPE, UFRJ, CP: 68502, CEP: 21945-970, Rio de Janeiro, RJ. E-mail: pinto@peq.coppe.ufrj.br 
oferecida por esses novos catalisadores, deve-se investigar e desenvolver mecanismos que evitem tal inconveniente. Um dos métodos mais empregados na indústria é a pré-polimerização, que consiste na polimerização da partícula de catalisador em condições brandas. Embora este processo permita o controle da morfologia da partícula de polímero em crescimento e evite o aparecimento de pontos quentes no interior do reator, ainda não foi completamente estudado e compreendido.

Sabe-se que o fenômeno dominante nos instantes iniciais da polimerização é a fragmentação, que consiste na quebra do suporte original em pequenas partículas. Entretanto, isto pode ocorrer de forma descontrolada, formando partículas muito pequenas e com morfologia irregular, resultando também em sérios problemas operacionais.

Embora seja fundamental para a continuidade da polimerização, a fragmentação ainda não foi totalmente elucidada. Sua observação experimental é muito difícil, pois ocorre em questão de segundos e num nível submicroscópico, havendo poucos trabalhos publicados na literatura ${ }^{[3]}$. Recentemente, Pater et al. ${ }^{[4]}$ fizeram um avanço considerável na investigação experimental. Eles montaram um pequeno reator para realizar a polimerização em lama do propileno a baixas taxas de reação, permitindo o estudo sistemático das partículas formadas. O rendimento da prépolimerização variou de 0,3 a 50 gramas de polipropileno por grama de catalisador empregado. A morfologia das partículas de pré-polímero assim produzidas foi analisada em microscópio eletrônico de varredura, esclarecendo alguns aspectos do processo de ruptura do suporte e crescimento do polímero.

Por outro lado, ainda não há métodos analíticos conclusivos que descrevam apropriadamente a fragmentação e possam ser usados para inferir como conduzir a reação de forma a se obter pré-polímero com as propriedades desejadas. Os modelos matemáticos utilizados para simular os processos de polimerização de olefinas admitem geralmente que a partícula de catalisador fragmenta-se de forma homogênea e controlada logo no início da reação, sendo que o tempo necessário para que a quebra do suporte se complete, da ordem de segundos ou menos, é neglicenciado. Esta hipótese é a base do modelo multigranular ${ }^{[5-7]}$ e do modelo do fluxo polimérico ${ }^{[8,9]}$. Após uma análise cuidadosa da lite- ratura científica, pode-se afirmar que apenas a série de trabalhos desenvolvidos por Chiovetta e colaboradores ${ }^{[10-12]}$ tentou construir um modelo matemático que mostrasse a importância da etapa de fragmentação sobre o restante da polimerização; ou seja, o efeito de um breve período de poucos segundos sobre o restante da reação, que pode levar horas. Isto significa que a maioria dos modelos existentes na literatura não é adequada para a representação do estágio inicial da polimerização de olefinas via catálise heterogênea.

Este trabalho propõe o desenvolvimento de uma metodologia baseada em modelo matemático que permite qualificar a morfologia das partículas resultantes da ruptura do catalisador suportado durante os estágios iniciais da reação de polimerização. A idéia central do método baseia-se na verificação da capacidade da partícula em dissipar energia resultante do acúmulo de tensões em seu interior durante o crescimento. Esta análise é feita por intermédio de um balanço de energia entre as quantidades acumulada e dissipada. Para tal, um modelo dinâmico da reação de pré-polimerização de olefinas ${ }^{[13]}$ é utilizado para calcular as grandezas envolvidas.

\section{A Fragmentação do Catalisador Suportado}

Os catalisadores organometálicos suportados em material inorgânico poroso (normalmente sílica ou cloreto de magnésio) constituem usualmente as partículas de catalisador empregadas na polimerização de olefinas em meio heterogêneo. Ao serem introduzidas num ambiente reacional, as partículas de catalisador entram em contato com as moléculas de monômero, determinando o início da reação de polimerização. As moléculas de monômero são adsorvidas pelos sítios ativos presentes na superfície e no interior da partícula. À medida que a reação avança, a expansão da fase polimérica leva a um acúmulo de tensão mecânica no interior da partícula, que pode resultar na ruptura ou não do suporte em consideração ${ }^{[14]}$. A quebra do suporte original em partículas menores aumenta a área específica e porosidade da partícula de catalisador, sendo fundamental para a continuidade da polimerização e para a estrutura final do polímero. Conhecendo-se o mecanismo de fragmentação da partícula durante os estágios inicias da polimerização, pode-se inferir a respeito do curso da reação, bem como estimar a morfologia da partícula de polímero produzida. 
Admitindo que o acúmulo de tensões é que provoca a quebra do suporte, a modelagem matemática proposta fundamenta-se em analisar a capacidade da partícula em dissipar a energia acumulada, por intermédio de um balanço de energia entre as quantidades dissipada e acumulada.

Considerando-se que uma partícula de catalisador suportado seja uma calota esférica isotrópica de volume $d V$, a energia $E$ acumulada por causa do acúmulo de tensões pode ser dada por:

$$
d E=p \cdot d V
$$

onde $p$ é a pressão interna resultante do acúmulo de polímero nos canais da partícula de catalisador. Portanto, a taxa de geração de energia na calota esférica de espessura $\Delta r$ é:

$$
\frac{d E}{d t}=4 \pi \cdot r^{2} \cdot \Delta r \cdot \frac{\partial p}{\partial t}
$$

Admitindo-se que uma equação de estado pode ser usada para avaliar a dependência da concentração mássica do material polimérico $(P)$ com a pressão e a temperatura:

$$
\frac{\partial P}{\partial t}=\left(\frac{\partial P}{\partial p}\right)_{T} \frac{\partial p}{\partial t}+\left(\frac{\partial P}{\partial T}\right)_{p} \frac{\partial T}{\partial t}
$$

Contudo, admitindo-se que a variação de pressão é dominada pelos efeitos da variação da densidade do material no interior da partícula, é razoável que o segundo termo seja desprezado, chegando-se a:

$$
\frac{\partial p}{\partial t}=\left(\frac{\partial p}{\partial P}\right)_{T} \frac{\partial P}{\partial t}
$$

que substituída na Equação (2), fornece a seguinte expressão para a taxa de geração de energia:

$$
\frac{d E}{d t}=4 \pi \cdot r^{2} \cdot \Delta r \cdot\left(\frac{\partial p}{\partial P}\right)_{T} \frac{\partial P}{\partial t}
$$

Observa-se que:

$$
\left(\frac{\partial p}{\partial P}\right)_{T}=K
$$

é o módulo de compressão específico do material polimérico. Por outro lado,

$$
\frac{\partial P}{\partial t}=R_{p}
$$

é reconhecido como a taxa de polimerização. Substituindo estas duas últimas expressões na Equação (5), obtém-se:

$$
\frac{d E}{d t}=4 \pi \cdot r^{2} \cdot \Delta r \cdot K \cdot R_{p}
$$

que relaciona a variação temporal de energia acumulada no interior da partícula com a quantidade de polímero produzido $\left(R_{p}\right)$, levando-se em consideração as características particulares do polímero $(K)$ por unidade de volume numa determinada calota esférica.

A fim de alcançar alta atividade de polimerização, a partícula de catalisador deve ser escolhida (material do suporte, tamanho da partícula, porosidade, etc.) de forma a garantir que a fragmentação ocorra logo no início da reação. Sendo assim, as partículas não devem ser capazes de acumular uma quantidade significativa de energia. Na verdade, a energia gerada deve ser de alguma forma dissipada pela partícula. Espera-se que o meio tenha capacidade de dissipar energia, ou na forma de ruptura do suporte, ou de deformação da massa polimérica. Admitindo-se que a capacidade de dissipar energia dependa da quantidade de polímero agregado à partícula original, tem-se:

$$
\frac{d E_{d}}{d t}=f(P) \cdot 4 \pi \cdot r^{2} \Delta r
$$

onde $f(P)$ caracteriza a capacidade da partícula em dissipar energia. Para a partícula original, $f(P)$ representa a resistência à tração ou compressão do suporte. Quando a partícula está repleta de polímero, $f(P)$ assemelha-se à resistência ao impacto deste material, pois é razoável supor que a massa polimérica tenha capacidade muito maior de dissipar energia que o suporte catalítico.

Sendo o meio incompressível ( $K$ muito grande), a estrutura isotrópica da partícula faz com que a energia seja dissipada na calota esférica de espessura $R$ $r$, que circunda o ponto de acúmulo de energia $(r)$, ou seja, onde está ocorrendo a quebra do suporte. A saber, $R$ é o raio da partícula. Sendo assim, o total de energia dissipada nessa calota é dado por:

$$
\left.\frac{d E_{d}}{d t}\right|_{I}=\int_{r}^{R} f(P) \cdot 4 \pi \cdot \xi^{2} d \xi
$$

Cabe ressaltar que a Equação (9) representa a energia dissipada numa camada de espessura infinitesimal, enquanto a Equação (10) fornece o to- 
tal de energia dissipada em toda a calota esférica que circunda o ponto de acúmulo de tensões.

Comparando-se as Equação (8) e (10), finalmente, define-se um critério que identifica os diferentes mecanismos de fragmentação e permite fazer inferências a respeito da evolução morfológica das partículas de polímero em crescimento.

a) $\mathrm{Se}$

$$
r^{2} \cdot \Delta r \cdot K \cdot R_{p}<\int_{r}^{R} f(P) \cdot \xi^{2} d \xi
$$

a partícula fragmenta-se, mas se mantém íntegra. A capacidade de dissipar energia na calota que circunda o ponto de acúmulo de tensões é maior que a taxa de geração. Além disso, dependendo da comparação entre a taxa local de geração de polímero $\left(K . R_{p}\right)$ e a capacidade local de dissipação de energia $(f(P))$, é possível identificar ainda duas situações distintas: a.1) $\mathrm{Se}$

$$
K \cdot R_{p}<f(P)
$$

a partícula fragmenta-se apenas localmente e a frente de dissipação de energia não se propaga, pois a taxa local de geração é menor que a capacidade local de dissipação.

a.2) $\mathrm{Se}$

$$
K \cdot R_{p}>f(P)
$$

a frente de dissipação de energia propaga-se e ocorre a deformação da partícula, pois a taxa local de geração é maior que a taxa local de dissipação. A partícula como um todo, no entanto, mantém-se íntegra.

b) $\mathrm{Se}$

$$
r^{2} \cdot \Delta r \cdot K \cdot R_{p}>\int_{r}^{R} f(P) \cdot \xi^{2} d \xi
$$

a partícula desintegra-se, pois a capacidade de dissipar energia na calota que circunda o ponto de acúmulo de tensões é menor que a taxa de geração. Dependendo da diferença relativa entre os termos, pode inclusive aparecer finos no reator de polimerização.

Portanto, esta análise evidencia que as características do suporte, do polímero e as condições da reação de polimerização interagem fortemente, determinando o momento da fragmentação e a morfologia das partículas de polímero produzidas.

Para que as equações acima sejam resolvidas, é imprescindível que $\Delta r$ seja definido. Sabendo-se que:

$$
\frac{\partial p}{\partial r}=\frac{\partial p}{\partial P} \cdot \frac{\partial P}{\partial r}
$$

e admitindo-se que $\partial p / \partial r$ pode ser aproximado por $\Delta p / \Delta r$ e identificando $K$ na expressão acima, tem-se:

$$
\Delta r=-\frac{\Delta p}{K \cdot\left(\frac{\partial P}{\partial r}\right)}
$$

Por sua vez, $\Delta p$ pode ser associado à pressão máxima $p_{\max }$ que o suporte pode resistir antes de se quebrar (resistência à tração). Portanto,

$$
\Delta r=\frac{p_{\max }}{K \cdot\left(\frac{\partial P}{\partial r}\right)}
$$

O termo $\partial P / \partial r$ indica a variação da concentração interna de polímero ao longo da direção radial. Caso $\partial P / \partial r$ seja nulo, deve-se aplicar uma aproximação de segunda ordem:

$$
\Delta r=\sqrt{\frac{2 p_{\max }}{K \cdot\left(\frac{\partial^{2} P}{\partial r^{2}}\right)}}
$$

O critério aqui definido permite a definição de dois índices que completam a caracterização da fragmentação: o comprimento da frente de dissipação $\left(\Delta r_{d}\right)$ e o índice de deformação (ID).

Caso a taxa local de geração seja menor que a capacidade local de dissipação, a frente de dissipação não se propaga, logo $\Delta r_{d}$ é nulo. Caso contrário, $\Delta r_{d}$ é calculado, considerando a igualdade da Equação (11). Para tal, basta substituir $R$ por $r+\Delta r_{d}$ e resolver a equação resultante para $\Delta r_{d}$ :

$$
\int_{r}^{r+\Delta r_{d}} f(P) \cdot \xi^{2} d \xi=r^{2} \cdot \Delta r \cdot K \cdot R_{p}
$$

De forma análoga ao caso anterior, se a taxa local de geração for menor que a capacidade local de dissipação, não há deformação da partícula de polímero, logo ID é nulo. Caso contrário, ID é calculado tomando-se a razão entre a quantidade de energia gerada e a quantidade de energia dissipada:

$$
I D=\frac{r^{2} \cdot \Delta r \cdot K \cdot R_{p}}{\int_{r}^{R} f(P) \cdot \xi^{2} d \xi}
$$




\section{Modelo Dinâmico de Pré-polimerização}

As grandezas envolvidas no desenvolvimento deste modelo, tais como $r, R_{p}, P$, e $\partial P / \partial r$, são calculadas resolvendo-se um modelo dinâmico ${ }^{[13]}$ que descreve a polimerização dentro da partícula de catalisador nos instantes iniciais da reação. Este modelo baseia-se no processo de reação com difusão, normalmente aceito para catálise heterogênea. O sistema composto por um conjunto de equações diferenciais parciais está apresentado a seguir:

$$
\begin{aligned}
& \frac{\partial M}{\partial t}=\frac{1}{r^{2}} \cdot \frac{\partial}{\partial r}\left(D_{M} \cdot r^{2} \cdot \frac{\partial M}{\partial r}\right)-R_{p} \\
& \frac{\partial P}{\partial t}=R_{p} \\
& \frac{\partial S}{\partial t}=\frac{1}{r^{2}} \cdot \frac{\partial}{\partial r}\left(D_{S} \cdot r^{2} \cdot \frac{\partial S}{\partial r}\right) \\
& \rho_{P} \cdot C_{P} \cdot \frac{\partial T}{\partial t}=\frac{1}{r^{2}} \cdot \frac{\partial}{\partial r}\left(k_{e} \cdot r^{2} \cdot \frac{\partial T}{\partial r}\right)-\Delta H_{p} \cdot R_{p} \\
& t=0, \quad \forall r: \quad M=P=S=0 \\
& I=I_{0} ; \quad T=T_{p o} \\
& t>0, \quad r=0: \quad \frac{\partial M}{\partial r}=\frac{\partial S}{\partial r}=0 \\
& \left.\frac{\partial P}{\partial t}\right|_{r=0}=R_{p} \\
& \frac{\partial T}{\partial r}=0 \\
& r=R: D_{M} \cdot \frac{\partial M}{\partial r}=k_{M} \cdot\left(M_{b}-M\right) \\
& D_{S} \cdot \frac{\partial S}{\partial r}=k_{S} \cdot\left(S_{b}-S\right) \\
& \left.\frac{\partial P}{\partial t}\right|_{r=R_{\text {cut }}}=R_{p} \\
& k_{e} \cdot \frac{\partial T}{\partial r}=h\left(T_{b}-T\right)
\end{aligned}
$$

e a restrição termodinâmica:

$$
\frac{M}{\rho_{M}}+\frac{P}{\rho_{P}}+\frac{S}{\rho_{S}}+\frac{I}{\rho_{I}}=1
$$

onde $M$ é a concentração mássica de monômero como função do tempo $(t)$ e do raio $(r) ; P$ é a concentração mássica do polímero; $S$ é a concentração mássica de solvente; $I$ é a concentração mássica de inerte; $T$ é a temperatura; $R_{p}$ é a taxa de polimerização; $\Delta H_{p}$ é o calor de polimerização; $\rho_{j}$ é a densidade da espécie $j$ em consideração; $C_{p}$ é a capacidade calorífica do meio; $D_{j}$ é a difusividade da espécie $j$ em consideração; $k_{M}$ é o coeficiente de transferência de massa do monômero no filme externo; $k_{S}$ é o coeficiente de transferência de massa do solvente no filme externo; $k_{e}$ é condutividade térmica da partícula; $h$ é o coeficiente de transferência de calor no filme externo; $M_{b}$ é a concentração mássica de monômero no meio reacional; $S_{b}$ é a concentração mássica de solvente no meio reacional; $I_{0}$ é a concentração mássica inicial de inerte na partícula de catalisador; $T_{b}$ é a temperatura da reação; $T_{p 0}$ é a temperatura inicial da partícula de catalisador; e $R$ é o raio da partícula de catalisador.

Este sistema é reduzido a um conjunto de equações diferenciais ordinárias pela aplicação do método da colocação ortogonal. Em seguida é executada a integração numérica no tempo. Assim, a concentração interna de polímero é calculada ao longo da reação e usada para estimar o momento da fragmentação, que é considerado como o instante em que a concentração de polímero torna-se igual à densidade esperada para o polímero nas condições de operação consideradas. Para este instante, também é calculado o perfil de concentração ao longo do raio da partícula.

Por outro lado, as grandezas $p_{\max }, f(P), K$ são características do suporte ou do polímero e podem ser obtidos na literatura ${ }^{[15]}$ ou avaliados com o auxílio de correlações termodinâmicas.

\section{Resultados e Discussão}

O modelo do processo de fragmentação foi aplicado ao processo de pré-polimerização do propileno em fase gasosa definido em trabalho anterior ${ }^{[13]}$. A Tabela 1 apresenta as condições operacionais e as principais propriedades térmicas, físicas e de transporte usadas nas simulações.

A fim de exemplificar a aplicação do modelo de pré-polimerização, a Figura 1 apresenta curvas típicas de concentração mássica de polímero, obtidas como resultado das simulações. É importante ressaltar que os perfis de polimerização mostrados na Figura 1 
indicam que a fragmentação pode começar em qualquer parte da partícula do catalisador e não somente na superfície, como normalmente aceito na literatura. A curva para $T_{b}=243 \mathrm{~K}$ atingiu a densidade esperada para o polímero $\left(0,905 \mathrm{~g} / \mathrm{cm}^{3}\right)$ no centro da partícula. Já a curva para $T_{b}=403 \mathrm{~K}$ somente atingiu a densidade esperada na superfície. Estes resultados de simulação são suportados por dados experimentais obtidos por Pater et al. ${ }^{[4]}$. Estes autores mostraram que o modelo de fragmentação normalmente aceito, que assume quebra do suporte camada por camada da superfície para o centro da partícula ${ }^{[10]}$ não é válido em certas condições. Portanto, o mecanismo camada por camada pode ser considerado como um caso particular do fenômeno de fragmentação.

Tabela 1. Condições operacionais, propriedades físicas, cinéticas, e de transporte para a pré-polimerização do propileno em fase gasosa.

\begin{tabular}{|c|c|}
\hline Propriedade & \\
\hline$M_{b}\left(\mathrm{~g} / \mathrm{cm}^{3}\right)$ & 0.042 \\
\hline$T_{b}(\mathrm{~K})$ & $240-343$ \\
\hline$T_{p 0}(\mathrm{~K})$ & $240-343$ \\
\hline$S_{b}\left(\mathrm{~g} / \mathrm{cm}^{3}\right)$ & 0 \\
\hline$p(\mathrm{~atm})$ & 21 \\
\hline$v(\mathrm{~cm} / \mathrm{s})$ & 2 \\
\hline $\mathrm{R}(\mathrm{cm})$ & $10^{-4}-10^{-1}$ \\
\hline$-\Delta H_{p}(\mathrm{cal} / \mathrm{g})$ & 590,5 \\
\hline$C_{p}(\mathrm{cal} / \mathrm{g} . \mathrm{K})$ & 0,331 \\
\hline$E_{343}(\mathrm{kcal} / \mathrm{gmol})$ & 10 \\
\hline$k_{p}(1$ cat/gmol sitio.s $)$ & 2640 \\
\hline$C^{*}$ (gmol sitio/l cat) & $10^{-3}$ \\
\hline$k_{e}(\mathrm{cal} / \mathrm{cm} \cdot \mathrm{s} \cdot \mathrm{K})$ & $2,6 \times 10^{-4}$ \\
\hline$k_{f}(\mathrm{cal} / \mathrm{cm} \cdot \mathrm{s} \cdot \mathrm{K})$ & $5 \times 10^{-5}$ \\
\hline${ }_{\mathrm{h}}\left(\mathrm{cal} / \mathrm{cm}^{2} \cdot \mathrm{s} \cdot \mathrm{K}\right)$ & 84,5 \\
\hline$k_{m}(\mathrm{~cm} / \mathrm{s})$ & 0,65 \\
\hline$\rho_{M}\left(\mathrm{~g} / \mathrm{cm}^{3}\right)$ & 0,042 \\
\hline$\rho_{P}\left(\mathrm{~g} / \mathrm{cm}^{3}\right)$ & 0,905 \\
\hline$\mu_{M}(\mathrm{cp})$ & 0,01 \\
\hline$D_{M}\left(\mathrm{~cm}^{2} / \mathrm{s}\right)$ & $5 \times 10^{-3}$ \\
\hline$\varepsilon$ & 0,4 \\
\hline$\tau$ & 4 \\
\hline
\end{tabular}

Onde $v$ é a velocidade do gás; $E_{343}$ é a energia de ativação; $k_{f}$ é a condutividade térmica do fluido; $\mu_{M}$ é a viscosidade do monômero; $\varepsilon$ é a porosidade; e $\tau$ é o fator de tortuosidade.

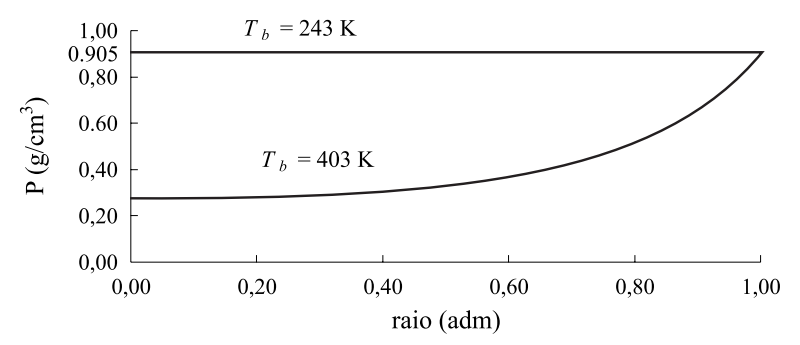

Figura 1. Perfil de concentração mássica de polímero numa partícula de catalisador com raio igual a $25 \mu \mathrm{m}$, no momento da fragmentação para duas temperaturas de operação diferentes.

Mais detalhes a respeito do perfil da concentração mássica de polímero podem ser verificadas em trabalho anterior ${ }^{[13]}$.

Além deste importante resultado, o modelo dinâmico calcula as grandezas $r, R_{p}, P$, e $\partial P / \partial r$ nos instantes iniciais da reação de pré-polimerização, tornando possível a análise definida neste trabalho, que será exemplificado adiante.

A Tabela 2 apresenta os valores encontrados na literatura ${ }^{[15]}$ para grandezas $p_{\max }, f(P), K$, completando os dados necessários para utilização do critério dos mecanismos de fragmentação. Cabe ressaltar que foi escolhida a sílica como suporte.

Realizando diversas simulações variando-se a temperatura de reação e o tamanho das partículas e aplicando o critério dos mecanismos de fragmentação, foi possível organizar os resultados, conforme está apresentado na Tabela 3. $T_{\text {int }}$ representa a temperatura limite do reator para a qual a partícula de um determinado tamanho mantém-se íntegra, de acordo com a Equação (12). Por sua vez, $T_{\text {frag }}$ representa a menor temperatura do reator para a qual a partícula desintegra-se, levando-se em consideração a Equação (14). No intervalo de temperatura entre $T_{i n t}$ e $T_{\text {frag }}$, a partícula comporta-se com o estabelecido no critério da Equação (13), ou seja, ocorre a deformação da partícula. Em todos os casos a concentração mássica de monômero no reator foi considerada igual a $0,042 \mathrm{~g} / \mathrm{cm}^{3}$.

De acordo com os resultados da Tabela 3, as partículas com raio igual a 1 e $10 \mu \mathrm{m}$ não apresentaram $T_{\text {frag }}$, ou seja, nas condições operacionais empregadas não correm o risco de desintegração. Para estes

Tabela 2. Propriedades mecânicas da sílica e do polipropileno.

\begin{tabular}{cc}
\hline Propriedade & \\
\hline$\rho_{\max }\left(\mathrm{N} / \mathrm{cm}^{2}\right)$ & 50 \\
$f(P)\left(\mathrm{N} / \mathrm{cm}^{2} . \mathrm{s}\right)$ & 101,6 \\
$K(\mathrm{~N} . \mathrm{cm} / \mathrm{g})$ & $3,8 \times 10^{5}$ \\
\hline
\end{tabular}


dois tamanhos de partícula, a maior temperatura do reator testada foi $423 \mathrm{~K}$. Mesmo para esta temperatura, as partículas continuariam apenas a se deformar, sem a ocorrência da desintegração. Cabe lembrar que os reatores de polimerização não operam neste patamar de temperatura, pois é muito próximo da temperatura de fusão do polipropileno. Além disso, observa-se que as partículas com tamanho menor que $100 \mu \mathrm{m}$ têm $T_{\text {frag }}$ menor ou igual a $326 \mathrm{~K}$. Sendo assim, pode-se dizer que é segura a operação do reator com esta faixa de tamanho de partículas. Por outro lado, as partículas com tamanho acima de $500 \mu \mathrm{m}$ podem oferecer sérios riscos à operação das plantas de polimerização. Isso porque, segundo os resultados obtidos, as partículas se desintegram a partir de $270 \mathrm{~K}$, provavelmente levando à formação de finos e instabilidade térmica no reator.

Os dados da Tabela 3 estão apresentados também na Figura 2. Esta figura sintetiza o critério desenvolvido neste trabalho e permite uma consulta fácil a respeito do mecanismo de fragmentação, definindo três regiões a saber: partícula íntegra, partícula deformada e partícula desintegrada. Por exemplo, para inferir a respeito do mecanismo de quebra, bastaria conhecer a distribuição do tamanho das partículas e a temperatura de operação. Sendo a temperatura do reator igual a $300 \mathrm{~K}$ e o tamanho médio das partículas de catalisador igual a $75 \mu \mathrm{m}$, consultando-se a figura 2 verifica-se facilmente que a operação é segura com a formação de partículas de polímero não desintegradas. Por outro lado, a figura 2 mostra qual a faixa de temperatura e tamanho de partícula que

Tabela 3. Organização dos resultados das simulações segundo o critério de fragmentação.

\begin{tabular}{ccc}
\hline Raio $(\mu \mathrm{m})$ & $T_{\text {int }}(\mathrm{K})$ & $T_{\text {frag }}(\mathrm{K})$ \\
\hline 1 & 243 & - \\
10 & 243 & - \\
25 & 243 & 398 \\
50 & 243 & 359 \\
75 & 243 & 338 \\
100 & 243 & 326 \\
250 & 242 & 291 \\
500 & 242 & 270 \\
750 & 241 & 260 \\
1000 & 240 & 253 \\
\hline
\end{tabular}

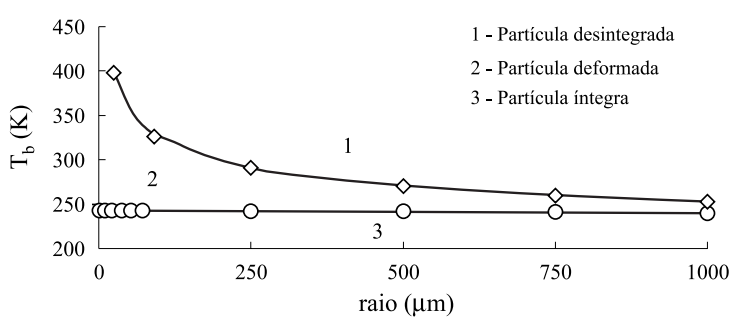

Figura 2. Critério para determinação do mecanismo de fragmentação em função da temperatura de reação e do tamanho da partícula de catalisador.

não devem ser empregados, a fim de evitar um polimerização problemática, com a obtenção de finos no reator e provavelmente instabilidade térmica no reator de polimerização.

É importante salientar que a figura 2 não é a única forma de apresentação do critério de controle morfológico apresentado. Estruturas similares podem ser construídas, por exemplo, a partir de variações da concentração de monômero no reator e de variações de concentração de catalisador suportado no interior da partícula. Dessa forma, é possível fazer um projeto consistente da partícula de catalisador, visando o adequado controle morfológico do polímero final.

\section{Conclusões}

Este trabalho propõe o desenvolvimento de uma metodologia que permite avaliar a morfologia das partículas resultantes da pré-polimerização do catalisador suportado. A idéia central do método baseia-se na verificação da capacidade da partícula em dissipar energia resultante do acúmulo de material em seu interior. O método necessita de grandezas que são calculadas via solução numérica de um modelo dinâmico e os parâmetros necessários são obtidos na literatura ou calculados com auxílio de correlações termodinâmicas.

Espera-se que o critério aqui desenvolvido e sintetizado em gráficos, como o apresentado na Figura 2 , seja de grande valia para a operação das plantas industriais de produção de poliolefinas, devido a sua grande simplicidade de utilização. Por outro lado, espera-se que o método seja importante também para ajudar a esclarecer o mecanismo da fragmentação nos estágios iniciais da polimerização.

\section{Agradecimentos}

Os autores agradecem o CNPq - Conselho Nacional de Pesquisas e Desenvolvimento Tecnológico e 
o PRONEX - Programa Nacional de Núcleos de Excelência pelo suporte financeiro.

\section{Referências Bibliográficas}

1. Kaminsky, W. - Journal of Chemical Society, Dalton Transactions, p.1413 (1998).

2. Xie, T.; Mcauley, K.; Hsu, J. \& Bacon, D. - Industrial Engineering Chemistry Research, 33, p.449 (1994).

3. Ferrero, M.; Sommer, R; Spanne, P.; Jones, K. \& Conner, W. - Journal of Polymer Science: Part A: Polymer Chemistry, 31, p.2507 (1993).

4. Pater, T.; Weickert, G.; Loos, J.; \& Swaaij, W. - Chemical Engineering Science, 56, p.4107 (2001).

5. Nagel, E.; Kirillov, V.\& Ray, W. - Ind. Eng. Chem. Prod. Res. Dev, 19, p.372 (1980).

6. Hutchinson, R.; Chen, C. \& Ray, W. - Journal of Applied Polymer Science, 44, p.1389 (1992).

7. McKenna, T. \& Soares, J. - Chemical Engineering Science, 56, p.3931 (2001).
8. Singh, D. \& Merrill, R. - Macromolecules, 4, p.559 (1971).

9. Schmeal W. \& Street, J. - AIChE Journal, 17, p.1188 (1971).

10. Laurence, R. \& Chiovetta, M. - Polymer Reaction Engineering, p.73 (1983).

11. Ferrero, M. \& Chiovetta, M. - Polymer Engineering and Science, 27, p.1436 (1987).

12. Estenoz, D. \& Chiovetta, M. - Polymer Engineering and Science, 36, p.2208 (1996).

13. Merquior, D.; Lima, E. \& Pinto, J. - "Modeling of Olefin Pre-Polymerization", in: Anais do $3^{\text {rd }}$ ENPROMER, p.919, Santa Fe, Argentina, Set (2001).

14. Webb, S.; Weist, E.; Chiovetta, M.; Laurence R. \& Conner, W. - The Canadian Journal of Chemical Engineering, 69, p.665 (1991).

15. Van Krevelen, D. - "Properties of Polymers", Elsevier Science, Amsterdam (1997).

Recebido: 10/08/2001

Aprovado: 24/07/2002 